\title{
The New EM: Specimen Preparation Procedures for Resin Embedding of Cryofixed Biological Samples in 6 Hours (Without a Microwave).
}

\author{
Kent L. McDonald ${ }^{1}$ \\ ${ }^{1}$ Electron Microscopy Laboratory, University of California, Berkeley, USA
}

Methods for embedding biological specimens into resin for subsequent thin sectioning were worked out over a decade or so from the late 1940's to early 1960's. By the mid to late 1960's the basic pattern was laid down that persists to this day as the most common way to prepare biological samples for imaging in a transmission electron microscope. This specimen preparation procedure consists of 3 sub-processes, fixation, dehydration and resin embedding, and typically takes more than 25 solution changes per sample and 3-4 days to complete. In the end the specimens are often distorted by artifacts unless the utmost care has been taken to optimize all the factors such as osmolarity, $\mathrm{pH}$, ionic composition, fixation time and temperature, etc., that can affect the cells. Even in the best cases there is likely to be shrinkage and extraction during dehydration. The current best practice to alleviate some of these problems is to cryoimmobilize cells and tissues (usually by high pressure freezing), dehydrate and chemically stabilize cell structures at low temperatures (freeze substitution), and embed in resin either by UV polymerization at low $\left(-25\right.$ to $\left.-50{ }^{\circ} \mathrm{C}\right)$ temperature, or polymerization at high $\left(60\right.$ to $\left.100{ }^{\circ} \mathrm{C}\right)$ temperatures. While the results from cryo-processing are clearly better than conventionals methods, the procedures often take even longer to complete. For the past three years or so we have been exploring ways to reduce the time that it takes to go from live cells to resin sections, as well as make the procedures simpler and reduce the need for some of the expensive equipment. We have successfully studied bacteria, ciliates, choanoflagellates, tissue culture cells, yeasts and other fungi, Drosophila embryos, C. elegans worms, zebrafish embryos, Arabidopsis pollen, and leaves of several different plants. We currently process most samples in about 6 hours. How this is done will be the subject of this talk, but some aspects are summarized below.

Fixation. Our preferred method for cryo-immobilization of cells is high pressure freezing, though alternative freezing strategies such as plunging into cryogen or impact onto a cooled metal block will work for some smaller samples.

Dehydration and chemical stabilization. This is done by a procedure called freeze substitution, in which cell water is replaced by organic solvent that may also contain fixatives such as osmium tetroxide, glutaraldehyde, and/or uranyl acetate. Our procedure [1] takes from 1 to 2.5 hours instead of the 2-3 days or more that it usually takes in most labs.

Resin inflitration and polymerization. We use increasing concentrations of resin/solvent mixtures $(25$, 50,75 , and $3 \times 100 \%$ ) to infiltrate with epoxy and methacrylate resins. But instead of leaving the specimens in each of these solutions for an hour or more as is usually done, we make an exchange every 5 minutes or so with agitation by rocking or rotating in between changes. We also concentrate the specimens by centrifugation at 2,000 x g for 30-60 seconds after each solution change.

In addition to preparing samples for morphological analysis by thin sections or by electron tomography of thicker sections, we have explored rapid methods for preparing cells for on-section immunogold labeling. We have found $[2,3]$ that freeze substitution in uranyl acetate/acetone only and embedding in LR White at room temperature will preserve ultrastructure sufficiently well for immuolabeling studies. The absence of the low concentrations of glutaraldehyde fixatives that are typically used for EM on-section immunolabeling studies means that we are likely to have better labeling efficiency with a wider range of antibodies. 
Results. For images of a variety of different cell types see [1-3]. Here we show images from materials that were prepared in about 5 hours following high pressure freezing. Figure 1 is from developing pollen in Arabidopsis thaliana. Pollen is considered a difficult specimen to embed but with our quick methods it seems to be just fine in spite of the pollen spore wall and the fact that we used Epon-Araldite, a very viscous resin. Figure 2 shows cross-sections of microvilli from the gut of a Caenorhabditis elegans worm with actin filaments labeled with $10 \mathrm{~nm}$ gold particles. This material was freeze-substituted in $0.2 \%$ uranyl acetate in acetone and embedded in LR White resin polymerized at $100^{\circ} \mathrm{C}$.

Summary. We have shown that it is possible to process many different types of samples into resin for thin section analysis in a fraction of the time that it usually takes. It is essential to start with well frozen material, though rapid resin embedding can be used for conventionally prepared samples if need be. Agitation during freeze substitution seems to be helpful for most samples, as well as for quick infiltration of resins. Finally, we have shown that freeze substitution without traditional fixatives can work well for on-section immunolabeling.
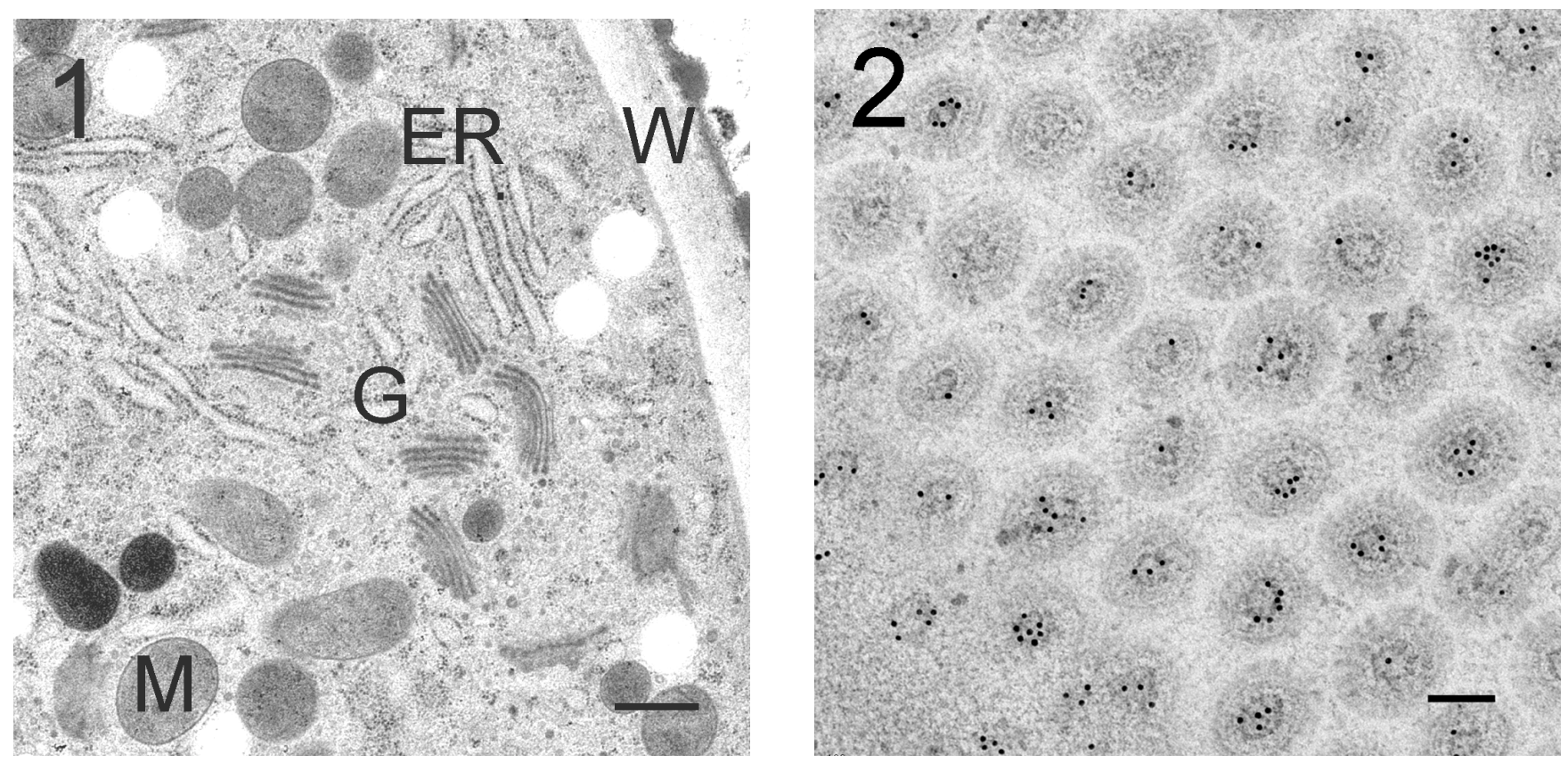

Figure Legends. Figure 1 shows a developing pollen grain of Arabidopsis embedded in Epon-Araldite. Mitochondria (M), endoplasmic reticulum (ER), and Golgi (G) are all well preserved and well infiltrated in spite of the thick pollen wall $(\mathrm{W})$. Bar $=0.5 \mu \mathrm{m}$. Figure 2 is of $C$. elegans gut microvilli in cross section labeled for actin with $10 \mathrm{~nm}$ gold on sections of LR White. Bar $=100 \mathrm{~nm}$.

\section{References}

[1] K. McDonald and R. Webb, J. Microscopy 243 (2011) p. 227.

[2] K. McDonald, Microsc. Microanal. doi:10.1017/S1431927613013846, Published online Nov. 19, 2013.

[3] K. McDonald, Protoplasma doi: 10.1007/s00709-013-0575-y, Published online Nov. 21, 2013

4) The author wishes to thank the following individuals for providing materials for this work: Michael Melzer, Leibnitz Institute of Plant Genetics and Crop Plant Research, Gatersleben, Germany for the Arabidopsis pollen, and Denise Lapidus and Barbara Meyer, University of California, Berkeley for the $C$. elegans worms. Thanks also to Richard Webb, Microscopy and Microanalysis Centre, University of Queensland, Australia for his continuing aid in the development of these rapid specimen preparation methods. 